\title{
Numerical integration of the interaction energy of two point charges
}

\author{
A. Gammal \\ Laboratório do Acelerador Linear, Instituto de Fsica, Universidade de São Paulo, C.P. 66.318, CEP 05389-970, \\ São Paulo, Brazil
}

Received 17 February 1997; accepted 26 February 1998

\begin{abstract}
We calculated numerically the energy interaction of two point charged particles. The integral has simple analytical result but is not easily solved numerically. Commercial programs as Mathematica $3.0^{\mathrm{TM}}$ and Maple $\mathrm{V}^{\mathrm{TM}}$ could not evaluate the integral and Gauss-Legendre method gave poor results. The method of Runge-Kutta integration was capable of solving it with good results. This integral showed to be a useful prototype for definite integration for double integration with poles. (C) 1998 IMACS/Elsevier Science B.V.
\end{abstract}

Keywords: Double integral; Poles; Point charges; Interaction energy

A very simple problem of electrostatics is to calculate analytically the energy stored by two point charges. But if we try to solve it numerically by integrating the fields we are faced with a very difficult problem of numerical integration of a double integral with poles. The Gauss-Legendre method [1] showed to be unsatisfactory for this problem and also the commercial programs Mathematica 3.0 ${ }^{\mathrm{TM}}[2]$ or Maple $\mathrm{V}^{\mathrm{TM}}$ could not solve it. The method of Runge-Kutta [3] succeeded as it is appropriate for treachery functions. We now proceed with the description of the analytical part and subsequently we show the numerical results.

The electric field $\mathbf{E}$ of a point charge $q$ can be described by [4]

$$
\mathbf{E}=\frac{q}{4 \pi \epsilon_{0} r^{2}} \hat{r}
$$

where $r$ is the radial distance, $\hat{r}=\mathbf{r} / r$ and $\epsilon_{0}$ is the dielectric constant. The energy stored in the field of a point charge in the volume $V$ is

$$
\mathscr{E}=\frac{\epsilon_{0}}{2} \int \mathbf{E}^{2} \mathrm{~d} V=\frac{1}{2} \frac{1}{(4 \pi)^{2} \epsilon_{0}} \int \frac{q^{2}}{r^{4}} \mathrm{~d} V
$$

that is named the self-energy of a point charge. 
If we have two point charges 1 and 2 separated by a distance of $2 a$, using the superposition principle, the resulting field will be

$$
\mathbf{E}=\mathbf{E}_{1}+\mathbf{E}_{2}
$$

The total energy stored in the field generated by the two charges is given by [4]

$$
\begin{aligned}
& \frac{\epsilon_{0}}{2} \int\left(\mathbf{E}_{1}+\mathbf{E}_{2}\right)^{2} \mathrm{~d} V=\frac{\epsilon_{0}}{2} \int\left(\frac{q_{1}}{4 \pi \epsilon_{0} r_{1}^{2}} \hat{r}_{1}+\frac{q_{2}}{4 \pi \epsilon_{0} r_{2}^{2}} \hat{r}_{2}\right)^{2} \mathrm{~d} V \\
& \quad=\frac{1}{2} \frac{1}{(4 \pi)^{2} \epsilon_{0}} \int\left(\frac{q_{1}^{2}}{r_{1}^{4}}+\frac{q_{2}^{2}}{r_{2}^{4}}+2 \frac{q_{1}}{r_{1}^{2}} \frac{q_{2}}{r_{2}^{2}} \hat{r}_{1} \cdot \hat{r}_{2}\right) \mathrm{d} V
\end{aligned}
$$

where $\hat{r}_{1}=\mathbf{r}_{1} / r_{1}$ and $\hat{r}_{2}=\mathbf{r}_{2} / r_{2}$.

Removing the self-energy of each charge we have the interaction energy

$$
\mathscr{E}_{\text {int }}=\frac{1}{2} \frac{1}{(4 \pi)^{2} \epsilon_{0}} \int\left(2 \frac{q_{1}}{r_{1}^{2}} \frac{q_{2}}{r_{2}^{2}} \hat{r}_{1} \cdot \hat{r}_{2}\right) \mathrm{d} V=\frac{1}{2} \frac{1}{(4 \pi)^{2} \epsilon_{0}} \int\left(2 \frac{q_{1}}{r_{1}^{3}} \frac{q_{2}}{r_{2}^{3}} \mathbf{r}_{1} \cdot \mathbf{r}_{2}\right) \mathrm{d} V
$$

In cylindrical coordinates, $\mathbf{r}_{1}=(\rho \cos \phi, \rho \sin \phi, z+a)$ and $\mathbf{r}_{2}=(\rho \cos \phi, \rho \sin \phi, z-a)$. The interaction energy (5) can be written as

$$
\mathscr{E}_{\text {int }}=\frac{1}{2} \frac{q_{1} q_{2}}{(4 \pi)^{2} \epsilon_{0}} 2 \pi \int_{-\infty}^{+\infty} \mathrm{d} z \int_{0}^{\infty} \rho \mathrm{d} \rho\left(2 \frac{\rho^{2}+z^{2}-a^{2}}{\left[\rho^{2}+(z+a)^{2}\right]^{3 / 2}\left[\rho^{2}+(z-a)^{2}\right]^{3 / 2}}\right)
$$

The interaction energy can also be calculated by the work to move the charge 2 to infinity, i.e.,

$$
\mathscr{E}_{\text {int }}=\text { Force } \times \text { displacement }=\int q_{2} \mathbf{E}_{1} \cdot \mathrm{d} \mathbf{l}=\int_{2 a}^{\infty} q_{2} \frac{q_{1}}{4 \pi \epsilon_{0} r^{2}} \mathrm{~d} r=\frac{q_{1} q_{2}}{4 \pi \epsilon_{0}} \frac{1}{2 a}
$$

comparing the results (6) with (7) we have

$$
\int_{-\infty}^{+\infty} \mathrm{d} z \int_{0}^{\infty} \rho \mathrm{d} \rho\left(\frac{\rho^{2}+z^{2}-a^{2}}{\left[\rho^{2}+(z+a)^{2}\right]^{3 / 2}\left[\rho^{2}+(z-a)^{2}\right]^{3 / 2}}\right)=\frac{1}{a}
$$

We can define

$$
I \equiv a \int_{-\infty}^{+\infty} \mathrm{d} z \int_{0}^{\infty} \rho \mathrm{d} \rho\left(\frac{\rho^{2}+z^{2}-a^{2}}{\left[\rho^{2}+(z+a)^{2}\right]^{3 / 2}\left[\rho^{2}+(z-a)^{2}\right]^{3 / 2}}\right)
$$

that is equal to 1 for whatever value of $a>0$, according to Eq. (8). 
Table 1

Numerical integration of $I$ with variable change $\rho_{u}=\rho /(1-\rho), \rho_{u} \in(0,1)$ and $z_{u}=z /(1-|z|), z_{u} \in(-1,1)($ exchanging the order of integration gave similar numerical results)

\begin{tabular}{rrlll}
\hline N.points $z$ & N.points $\rho$ & $I_{a=1}$ & $I_{a=10}$ & Delay \\
\hline 50 & 50 & 1.00908917858613 & 1.11237253285843 & $<1 \mathrm{~s}$ \\
100 & 100 & 0.972914740382022 & 0.924051121779205 & $<1 \mathrm{~s}$ \\
500 & 500 & 1.00908917858613 & 0.993471125547867 & $<1 \mathrm{~s}$ \\
1000 & 1000 & 0.997280353909560 & 1.00641014440464 & $15 \mathrm{~s}$ \\
5000 & 5000 & 1.00090710285742 & 1.00092943464365 & $5 \mathrm{~min}$ \\
\hline
\end{tabular}

Table 2

Numerical integration of the interaction energy of two point charges

\begin{tabular}{llll}
\hline eps & $I_{a=1}$ & $I_{a=10}$ & Delay \\
\hline $10^{-4}$ & 0.998299125109709 & 0.998449022955381 & $<1 \mathrm{~s}$ \\
$10^{-6}$ & 0.999993228949804 & 0.999996822852136 & $<1 \mathrm{~s}$ \\
$10^{-8}$ & 0.999999752816064 & 1.00000007700164 & $20 \mathrm{~s}$ \\
$10^{-10}$ & 0.999999999868884 & 0.999999997838525 & $2 \mathrm{~min}$ \\
\hline
\end{tabular}

Evaluation of $I$ by Runge-Kutta method with variable change $\rho_{u}=\rho /(1-\rho), \rho_{u} \in(0,1)$ and $z_{u}=z /(1-|z|), z_{u} \in(-1,1)$. The infinity value was taken as $\rho_{u}=\left|z_{u}\right|=1-10^{-10}$. First integrating in $\rho$ and later in $z$.

So although the integral of interaction energy looks a little cumbersome, it has a very simple analytical result. We tried to solve it by Mathematica $3.0^{\mathrm{TM}}$ and by Maple $\mathrm{V}^{\mathrm{TM}}$ by analytical and by numerical methods but we do not obtain the proper results. Also using the Gauss-Legendre method with routine gauleg. $f$ from [3], gave poor results as shown in Table 1.

As the function has poles it is considered as a treachery integral and in this case is better to use the Runge-Kutta method of integration [3]. We applied the Runge-Kutta method of integration with the subroutines odeint.f, rkck.f, rqck.f, derivs.f from [3]. Integrating first in $\rho$ and later in $z$ the method succeeded as shown in Table 2. The parameter eps is the step used in the method and the smaller the step better is the integration. The comparison for distance $a=1$ and $a=10$ show that the precision has little dependence on $a$. Surprisingly it could not integrate by exchanging the order of integration between $\rho$ and $z$.

Concluding, the energy interaction between two point charges generates a double integral with poles over the charge points. This integral has a simple analytical result. This integral can be used as a prototype for double integration with poles. Mathematica $3.0^{\mathrm{TM}}$ and Maple $\mathrm{V}^{\mathrm{TM}}$ could not solve the integral neither analytically nor numerically and Gauss-Legendre method gives poor results. RungeKutta method succeeded but only integrating first $\rho$ and later $z$.

The calculations were all made with double precision in a Digital ${ }^{\mathrm{TM}}$ alpha2000 server.

\section{Acknowledgements}

I thank Prof. T. Frederico and I.D. Goldman for useful discussions. 


\section{References}

[1] B. Carnahan, H.A. Luther, J.O. Wilkes, Digital Computing and Numerical Methods, Wiley, New York, 1969.

[2] S. Wolfram, The Mathematica Book, Cambridge University Press, Cambridge, 1991.

[3] W.H. Press, S.A. Teulkolsky, W.T. Vettering, B.P. Plannery, Numerical Recipes in Fortran, 2nd ed., Cambridge University Press, Cambridge, 1992.

[4] A.D. Jackson, Classical Electrodynamics, 2nd ed., Wiley, New York, 1975. 